\title{
A RESEARCH ON VOCABULARY TEACHING STRATEGIES AND STUDENTS' MASTERY
}

\author{
(C) Tian Yuan*, Liu Bingbing \\ School of Foreign Languages, Liaoning Shihua University \\ 113001 Fushun, Liaoning, China. \\ E-mail: timryan@163.com
}

\begin{abstract}
By means of questionnaire and quantitative research, this article aims at investigating the effects on students' mastery of vocabulary by studying teachers' adoption of seven kinds of common vocabulary teaching strategies and the usage of analyzing strategies in intensive English in order to improve vocabulary teaching strategies and to help enlarge students' vocabulary.

Keywords: vocabulary teaching, teaching strategies, students' mastery, teaching effect.
\end{abstract}

\section{Introduction}

Cohen (1990) and Nation (1990) elaborately discussed various strategies about vocabulary teaching, which include phonetics method, lexical approach, visual sense method, associative memory approach, contextual approach, inductive method and cultural meaning method. The research in this article is based on these vocabulary teaching strategies.

\section{Research Ways and Data Collection}

The subjects of this research are 171 sophomores of non-English major, including 145 boys and 26 girls. The questionnaire is mainly divided into vocabulary teaching methods and teaching effects. The method based on seven strategies, adopting from "teachers always use the method" to "teachers never use the method" and students also evaluate teachers' vocabulary teaching effect from "best" to "none". These questions constitute a form requiring the subjects to choose the congruous or approximate choices according to their own. Subtracting 14 useless questionnaires, we totally get 157 effective papers. The author used data analysis software to analyze the data.

\section{Research Effect and Discussion}

\subsection{Phonetics Method}

Teachers who always use this method account for $10 \%$, often use $52.5 \%$, sometimes use $25 \%$, seldom use $12.5 \%$, and never use $0 \%$.As to the effects, best makes up for $7.5 \%$, better $30 \%$, good $57.5 \%$, bad $5 \%$, and no effects $0 \%$. According to the data of feedback, teachers who often use phonetics as the teaching method account for $52.5 \%$, that is to say, more than half of teachers use this method, but $30 \%$ students think its effect is just better, and still $57.5 \%$ students think it's good.

\footnotetext{
* corresponding author
} 
Students don't understand how to use pronunciation rules to remember English words, so learning words by rote will not make their memory firm, thus it is a waste of time and inefficient.

\subsection{Lexical Approach}

Teachers who always use this method account for $7.5 \%$, often use $45 \%$, sometimes use $37.5 \%$, seldom use $10 \%$, and never use $0 \%$. As to the effects, best makes up for $5 \%$, better $27.5 \%$, good $60 \%$, bad $7 \%$, and no effects $0 \%$.The application of the rules of English vocabulary structure can not only help students understand the basic meaning but also achieve the purpose of enlarging vocabulary. $45 \%$ teachers often use this strategy, and $60 \%$ students think lexical approach is good for them to recite words. Lexical approach is an important task of vocabulary teaching, and we should pay more attention.

\subsection{Visual Sense Method}

Teachers who always use this method account for $10 \%$, often use $25 \%$, sometimes use $37.5 \%$, seldom use $27.5 \%$, and never use $0 \%$. As to the effects, best makes up for $0 \%$, better $15 \%$, good $60 \%$, bad $25 \%$, and no effects $0 \%$.Teacher always use this teaching method, but the effect is common. There are some limits like culture, experience, structure of knowledge, and region. Some concepts can hardly image themselves in students' minds, but teachers can use objects, pictures, screens, actions, gestures and non-verbal expressions to give students a deep expression.

\subsection{Associative Memory Approach}

Teachers who always use this method account for $7.5 \%$, often use $25 \%$, sometimes use $45 \%$, seldom use $17.5 \%$, and never use $5 \%$. As to the effects, best makes up for $12.5 \%$, better $32.5 \%$, good $45 \%$, bad $45 \%$, and no effects $0 \%$.From the result, teachers always use it, and students also like this method. Vocabulary is stored in different categories in one's memory, and it is a collection based on some relations, which will be forgotten over time. Associative memory approach will help make students remember new words efficiently, arouse their interest and enlarge vocabulary.

\subsection{Contextual Approach}

Teachers who always use this method account for $10 \%$, often use $32.5 \%$, sometimes use $47.5 \%$, seldom use $10 \%$, and never use $0 \%$. As to the effects, best makes up for $10 \%$, better $42.5 \%$, good $47.5 \%$, bad $0 \%$, and no effects $0 \%$.Teacher use this method more frequently, and the effect is very good. Situational language teaching requires teachers to give a context in order to let students grasp the meaning. The research effects indicate that this method can make students remember words exactly, and make them remember words as soon as they recall the scenes.

\subsection{Inductive Method}

Teachers who always use this method account for $10 \%$, often use $50 \%$, sometimes use $22.5 \%$, seldom use $32.5 \%$, and never use $0 \%$. As to the effects, best makes up for $17.5 \%$, better $35 \%$, good $30 \%$, bad $17.5 \%$, and no effects $0 \%$.Teachers often use this method, and it is very popular in students, so the effect is best. The research of Craik and Tulving (1975) indicated that the subjects who were 
taught to classify words performed better than those who were not. So as for vocabulary teaching, teachers should guide students to classify and handle with the words that are needed, in order to improve memory effect and enlarge vocabulary as soon as possible.

\subsection{Cultural Meaning Method}

Teachers who always use this method account for $0 \%$, often use $5 \%$, sometimes use $45 \%$, seldom use $50 \%$, and never use $0 \%$. As to the effects, best makes up for $2.5 \%$, better $30 \%$, good $55 \%$, bad $12.5 \%$, and no effects $0 \%$.Although teachers seldom put culture into vocabulary teaching, students' feedbacks are good. This demonstrates that students are willing to accept vocabulary with cultural knowledge. So during English vocabulary teaching, teachers should help students learn and grasp the concept meaning of vocabulary, at the same time cultivate their cultural consciousness gradually.

\section{Conclusion}

From the analysis above, we know that teachers in our university prefer associative memory approach and inductive approach to other teaching methods. Although these teaching strategies were proved efficiently, they are not paid much attention in classroom teaching. Hopefully, this article will help teachers realize this problem, and better use these strategies in vocabulary teaching.

\section{REFERENCES}

1. Cohen A. D. Language Learning. New York: Newbury House, 1990.

2. Craik F. I. M., E. Tulving. Depth of Processing and the Retention of Words in Episodic Memory // Journal of Experimental Psychology. 1975. V. 104. P. 268-294.

3. Nation I. S. P. Teaching and Learning Vocabulary. New York: Newbury House, 1990. 